ARTICLE

Received 2 Aug 2015 | Accepted 29 Jan 2016 | Published 8 Mar $2016 \quad$ DOl: 10.1038/ncomms10912 OPEN

\title{
Structural basis of allosteric and synergistic activation of AMPK by furan-2-phosphonic derivative $\mathrm{C} 2$ binding
}

Christopher G. Langendorf ${ }^{1, \star}$, Kevin R.W. Ngoei ${ }^{1, \star}$, John W. Scott ${ }^{1}$, Naomi X.Y. Ling ${ }^{2}$, Sam M.A. Issa ${ }^{1,2}$, Michael A. Gorman ${ }^{3}$, Michael W. Parker ${ }^{3}$, Kei Sakamoto ${ }^{4}$, Jonathan S. Oakhill ${ }^{2} \&$ Bruce E. Kemp ${ }^{1,5}$

The metabolic stress-sensing enzyme AMP-activated protein kinase (AMPK) is responsible for regulating metabolism in response to energy supply and demand. Drugs that activate AMPK may be useful in the treatment of metabolic diseases including type 2 diabetes. We have determined the crystal structure of AMPK in complex with its activator 5-(5-hydroxyl-isoxazol-3-yl)-furan-2-phosphonic acid (C2), revealing two C2-binding sites in the $\gamma$-subunit distinct from nucleotide sites. C2 acts synergistically with the drug A769662 to activate AMPK $\alpha 1$-containing complexes independent of upstream kinases. Our results show that dual drug therapies could be effective AMPK-targeting strategies to treat metabolic diseases.

\footnotetext{
${ }^{1}$ Protein Chemistry \& Metabolism, St Vincent's Institute of Medical Research, University of Melbourne, 41 Victoria Parade, Fitzroy, Victoria 3065, Australia. ${ }^{2}$ Metabolic Signaling Laboratory, St Vincent's Institute of Medical Research, University of Melbourne, 41 Victoria Parade, Fitzroy, Victoria 3065, Australia. ${ }^{3}$ ACRF Rational Drug Discovery Centre, St Vincent's Institute of Medical Research, University of Melbourne, 41 Victoria Parade, Fitzroy, Victoria 3065 , Australia. ${ }^{4}$ Nestlé Institute of Health Sciences SA, EPFL Innovation Park, bâtiment G, 1015 Lausanne, Switzerland. ${ }^{5}$ Mary MacKillop Institute for Health Research Australian Catholic University, Victoria Parade, Fitzroy, Victoria 3065, Australia. ${ }^{\star}$ These authors contributed equally to this work. Correspondence and requests for materials should be addressed to C.G.L. (email: clangendorf@svi.edu.au) or to B.E.K. (email: bkemp@svi.edu.au).
} 
A MP-activated protein kinase (AMPK) is a metabolic stress-sensing kinase responsible for regulating metabolism in response to energy supply and demand. During metabolic stress the cellular AMP/ATP ratio increases leading to activation of AMPK, which in turn switches off energyconsuming anabolic pathways and switches on catabolic pathways to restore ATP levels. The AMPK $\alpha \beta \gamma$ heterotrimer comprises a catalytic $\alpha$-subunit associated with $\beta$ and $\gamma$ regulatory subunits (Fig. 1a). Adenine nucleotides (ATP, ADP and AMP) bind interchangeably to the $\gamma$ subunit where three of the four cystathionine $\beta$-synthase (CBS) tandem-repeat sequences provide nucleotide-binding sites ${ }^{1}$. Allosteric activation of AMPK by AMP appears to involve all three sites but site 3 may be the most important ${ }^{2,3}$. AMP binding initiates AMPK signalling by promoting phosphorylation of AMPK on $\alpha$-Thr172 by

a

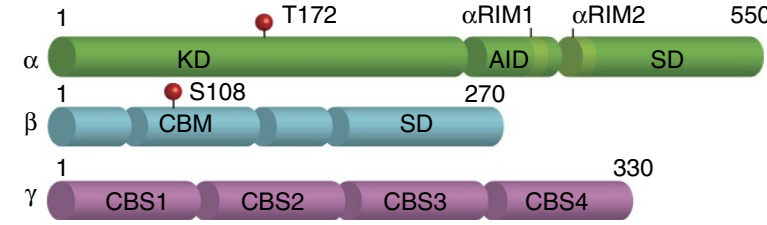

b

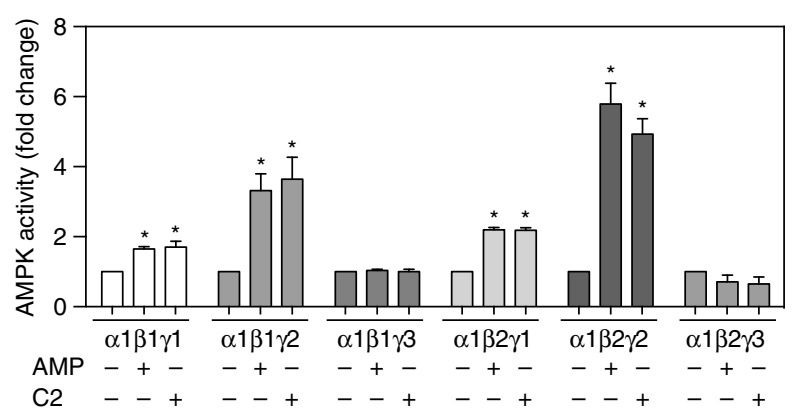

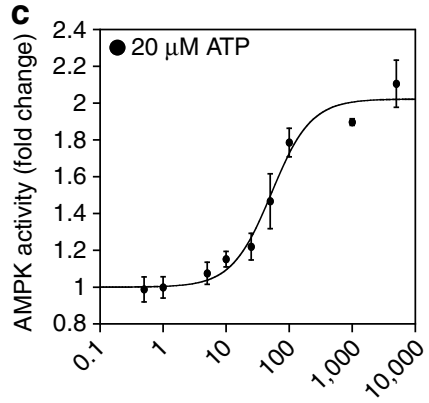

[C2] (nM)

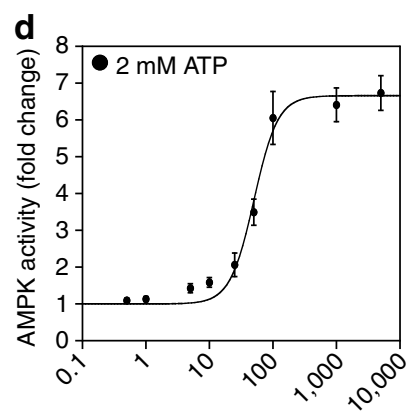

[C2] (nM)
Figure 1 | Allosteric activation of AMPK by C2. (a) Schematic representation of the three AMPK subunits: the catalytic $\alpha$-subunit, and the regulatory $\beta$ and $\gamma$-subunits. (b) C2 activation of AMPK $\alpha 1$ complexes. Activities of COS7 cell-expressed AMPK (complexes as indicated) were assayed in the absence or presence of $C 2(0.1 \mu \mathrm{M})$ or AMP $(100 \mu \mathrm{M})$. Results from triplicate experiments are presented as mean AMPK activity (fold change relative to basal \pm s.e.m.), with statistical tests conducted using one-way ANOVA. ${ }^{\star} P<0.05$ indicates significant increase in activity compared to untreated control. Effect of ATP concentration on C2 activation of AMPK $\alpha 1$. C2 dose-response $(0-5 \mu \mathrm{M})$ at (c) low $(20 \mu \mathrm{M})$ ATP and (d) near-physiological ( $2 \mathrm{mM}$ ) ATP. Results from six independent experiments are plotted as AMPK activity (fold change \pm s.e.m.) versus [C2] (nM). The values for $\mathrm{EC}_{50}$, fold stimulation and Hill coefficients were calculated based on fitting the data to the equation:

Activity $=$ Basal $+\left(\left((\right.\right.$ Fold stimulation $\times$ Basal $)-$ Basal $\left.\times\left[\mathrm{C}^{2}\right]^{\text {h }}\right) /$ $\left.\left(\left(E_{50}\right)^{\mathrm{h}}+[C 2]^{\mathrm{h}}\right)\right)$. AID, auto-inhibitory domain; CBM, carbohydratebinding module; $C B S$, cystathionine $\beta$-synthase motif; $K D$, kinase domain; RIM, regulatory subunit-interacting motif; SD, scaffold domain. upstream kinases LKB1 and $\mathrm{Ca}^{2+} /$ calmodulin-dependent protein kinase 2 (CaMKK2). Once phosphorylated on $\alpha$-Thr172 AMPK is further activated by AMP, and AMP sustains AMPK signalling by inhibiting dephosphorylation of $\alpha$-pThr172 (ref. 4). AMP stimulation of $\alpha$ Thr172 phosphorylation is dependent on $\mathrm{N}$-terminal myristoylation of the $\beta$-subunit, since we demonstrated that AMP stimulation by both upstream kinases is lost following $\beta-\mathrm{G} 2 \mathrm{~A}$ mutation ${ }^{2}$. ATP on the other hand opposes allosteric activation by AMP as well as promoting the dephosphorylation of $\alpha$-Thr172 (ref. 5). The $\alpha$-regulatory subunit-interacting motif $2(\alpha \text { RIM2; Fig. } 1 \mathrm{a})^{6}$ is required for sensing the adenine nucleotide bound state of the $\gamma$-subunit and transducing this signal to the $\alpha$-catalytic domain, resulting in either stimulation (AMP) or inhibition (ATP) of AMPK activity ${ }^{1,6-8}$.

There has been keen interest in developing AMPK-activating drugs for potential therapeutic use in treating metabolic diseases including type 2 diabetes, obesity and cardiovascular disease. A number of small-molecule activators have been identified ${ }^{9}$ and two of these, A769662 (Abbott Laboratories) and 991 (Merck Sharp and Dohme Corporation and Metabasis Therapeutics), were shown in milestone structures to bind to a site formed between the small lobe of the $\alpha$-subunit kinase domain and the $\beta$-subunit carbohydrate-binding module $(\mathrm{CBM})^{8,10}$ termed the allosteric drug and metabolite-binding site $(\mathrm{ADaM})^{11}$. Salicylate, the active metabolite of aspirin, is also thought to bind to this site $^{12}$. ADaM site stabilization is enhanced by phosphorylation of the $\beta$-subunit residue Ser108 (refs 13,14). We have shown that the need for $\beta$-pSer108 can be bypassed and that dephosphorylated AMPK (or dephosphorylation mimic $\alpha 1-\mathrm{T} 172 \mathrm{~A} \beta 1-\mathrm{S} 108 \mathrm{~A} \gamma 1$ mutant) can be activated synergistically by A769662 and AMP whereas there is negligible activity with either activator alone ${ }^{13}$.

Another potent small-molecule allosteric activator of AMPK, 5-(5-hydroxyl-isoxazol-3-yl)-furan-2-phosphonic acid (compound 2, C2, Metabasis Therapeutics), was identified by screening an AMP mimetic library ${ }^{15}$. It is thought that $\mathrm{C} 2$ activates both AMPK $\alpha 1$ and $\alpha 2$ isoforms in cell-free assays by binding to the $\gamma$-subunit. Although C2 is impermeable to cells, its corresponding di-iso-propyl phosphoester prodrug (compound 13, C13) preferentially stimulates AMPK $\alpha 1$ in mammalian cells $^{16}$. AMP and C2 likely share a common AMPK-activating mechanism as their allosteric effects are not additive, and sensitivity to both is lost in the $\gamma 2$-R531G AMP-insensitive mutant ${ }^{16}$. The purpose of our study was to identify the C2-binding site(s) on AMPK and investigate the relationship between activation of AMPK by A769662 and C2.

We have determined the crystal structure of the C2: AMPK complex, revealing two C2-binding sites in the $\gamma$-subunit distinct from nucleotide sites. C2 acts synergistically with the drug A769662 to activate AMPK $\alpha 1$-containing complexes independent of upstream kinases. Our results raise the possibility that dual drug therapies could be effective AMPK-targeting strategies to treat metabolic diseases.

\section{Results}

Allosteric activation of AMPK. We found that C2 activates both AMPK $\gamma 1$ and $\gamma 2$ complexes $(\alpha 1 \beta 1 \gamma 1$ and $\alpha 1 \beta 2 \gamma 1 ; \alpha 1 \beta 1 \gamma 2$ and $\alpha 1 \beta 2 \gamma 2$ ) to a similar degree as AMP, whereas neither C2 nor AMP-activated $\gamma 3$-containing complexes $(\alpha 1 \beta 1 \gamma 3$ and $\alpha 1 \beta 2 \gamma 3)$ (Fig. 1b). Lack of AMP activation of $\gamma 3$-containing complexes has also been reported recently ${ }^{17}$ but not in all studies ${ }^{18,19}$.

Since ATP concentration was previously shown to alter AMP dependence 5 , we investigated C2 activation of AMPK $\alpha 1$ at low $(20 \mu \mathrm{M})$ and physiological $(2 \mathrm{mM})$ ATP concentrations. Comparison of dose-response curves revealed that the half-maximal concentrations for $\mathrm{C} 2$ activation were similar at 
both low and high ATP $(52.3 \pm 7.9$ and $50.3 \pm 4.4 \mathrm{nM}$ for $20 \mu \mathrm{M}$ and $2 \mathrm{mM}$ ATP, respectively; Fig. 1c,d), implying that $\mathrm{C} 2$ and ATP binding is non-competitive. In contrast, previous studies have shown that the concentration of AMP required for halfmaximal activation increases with higher concentrations of ATP, which is consistent with AMP/ATP competition at the $\gamma$-subunit allosteric sites ${ }^{5}$. We also observed that $\mathrm{C} 2$ activation becomes strongly co-operative at high ATP, as shown by a shift in the Hill coefficient from $1.4 \pm 0.3$ ( $20 \mu \mathrm{M}$ ATP) to $2.3 \pm 0.4$ ( $2 \mathrm{mM} \mathrm{ATP})$ (Fig. 1c,d). In contrast, AMP does not exhibit co-operative binding at high ATP concentrations (Hill coefficient 1.0 \pm 0.2 ; Supplementary Fig. 1 and Supplementary Table 1). We are uncertain of the structural basis for the effect of ATP on C2 co-operative binding as well as the kinetic basis for ATP not influencing AMP activation in a co-operative manner. The $\gamma$-subunit resembles a classic multimeric-enzyme with its 4 CBS tandem repeats. One interpretation is that ATP binding to the $\gamma$-subunit influences $\mathrm{C} 2$ co-operativity but we have been unable to obtain crystals with both C2 and ATP bound to the $\gamma$-subunit thus far. In the event ATP cannot bind to $\gamma$ in the presence of C2 we cannot formally eliminate the possibility that ATP binding at the kinase active site $(\alpha$-subunit) rather than the $\gamma$-subunit may induce $\mathrm{C} 2$ co-operative binding.

Synergistic activation of AMPK. We examined whether C2, like AMP, can synergize with A769662 to activate dephosphorylated AMPK (Supplementary Fig. 2a). Furthermore, we explored whether this synergistic activation is also present in the AMPK $\alpha 2$ isoform. Modest allosteric activations were observed with $\lambda$-phosphatase pre-treated AMPK $\alpha 1$ or $\alpha 2$ complexes individually incubated with AMP or C2 or A769662 (Fig. 2a). We attribute the larger A769662 response using AMPK $\alpha 1$ to residual $\beta 1$-Ser108 phosphorylation persisting after $\lambda$-phosphatase treatment (Supplementary Fig. 2a). Synergistic activation occurred when the $\lambda$-phosphatase-treated AMPK $\alpha 1$ (basal activity

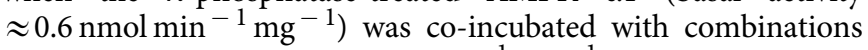
of AMP/A769662 ( $\approx 671 \mathrm{nmol} \mathrm{min}{ }^{-1} \mathrm{mg}^{-1}$ or $\approx 1100$-fold $)$ and C2/A769662 ( $\approx 636 \mathrm{nmol} \mathrm{min}^{-1} \mathrm{mg}^{-1}$ or $\approx 1040$-fold), but not with CaMKK2-treated AMPK $\alpha 1$ (Fig. 2a,b; Table 1). It should be noted the maximum activity observed in C2-stimulated, CaMKK2-treated AMPK $\alpha 1$ is $\approx$ threefold higher than the maximum activity observed with the $\lambda$-phosphatase-treated AMPK $\alpha 1$ co-incubated with C2/A769662. Moreover, the synergistic activation was specific to AMPK $\alpha 1$, as the $\lambda$-phosphatase-

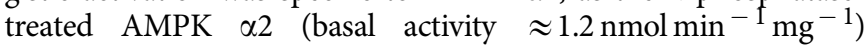
co-incubated with AMP/A769662 or C2/A769662 was relatively insensitive to dual ligand activation (AMP/A769662

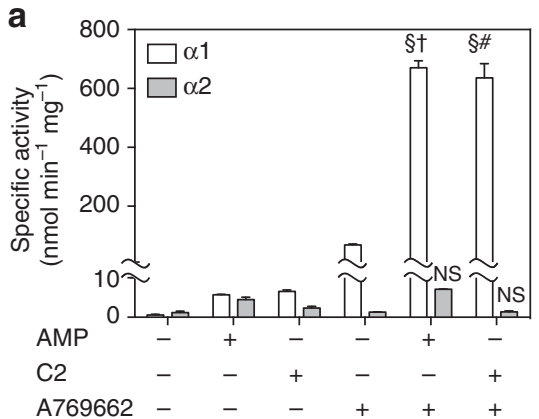

activation $\approx 7.2 \mathrm{nmol} \mathrm{min}^{-1} \mathrm{mg}^{-1}$ or $\approx 6.1$-fold; C2/A769662 activation $\approx 1.3 \mathrm{nmol} \mathrm{min}{ }^{-1} \mathrm{mg}^{-1}$ or $\approx 1.2$-fold; Fig. 2a; Table 1). Similarly to CaMKK2-treated AMPK $\alpha 1$, the CaMKK2treated AMPK $\alpha 2$ did not exhibit dual synergistic activation by both AMP/A769662 and C2/A769662 (Fig. 2b).

Novel C2-binding site in the $\gamma$-subunit. To gain further insight into the mechanism of $\mathrm{C} 2$-mediated AMPK activation we solved the X-ray crystal structure of full-length $\alpha 2 \beta 1 \gamma 1$ isoform cocrystallized with C2 and AMP to $2.99 \AA$ resolution (Table 2). The structure revealed two molecules of $\mathrm{C} 2$, bound within the solventaccessible core of the $\gamma$-subunit. The phosphate groups of both C2 molecules overlap the phosphate binding sites of AMP in sites 1 and 4, while the 5-(5-hydroxyl-isoxazol-3-yl)-furan moieties occupy novel binding sites independent from the AMP-binding sites (Fig. 3a-c; Supplementary Fig. 3a). The asymmetric unit contained two AMPK heterotrimers. One heterotrimer contained AMP bound at each of CBS sites 3 and 4 (Fig. 3a, white) whereas the other heterotrimer was devoid of AMP and fortuitously contained two molecules of $\mathrm{C} 2$ instead (Fig. 3a-c). The two distinct liganded complexes allowed us to comprehensively probe the differences between AMP and C2 binding to AMPK.

Our AMP-bound heterotrimer closely resembles the previously published AMP-bound $\alpha 2 \beta 1 \gamma 1$ structure (PDB 4CFE) ${ }^{8}$. The two molecules superpose with a r.m.s. deviation of $0.55 \AA$ over $884 \mathrm{C} \alpha$ atoms, with key AMP-interacting residues having the same conformation. In contrast, the C2-bound molecule has an r.m.s. deviation of $0.75 \AA$ (over $864 \mathrm{C} \alpha$ atoms) when superimposed with $4 \mathrm{CFE}^{8}$. The largest structural difference between the $\mathrm{C} 2$ and AMP-bound heterotrimer is in the $\beta$-CBM and the N-lobe of the kinase domain, where a shift of up to $4.0 \AA$ Accurs (Fig. 3a). This shift reflects the formation of a canonical kinase salt bridge, formed between conserved residues $\alpha$-Lys 45 and $\alpha$-Glu64, in the C2-bound heterotrimer. This important interaction is regarded as an active kinase conformation signature ${ }^{20}$ and is conspicuously absent in previous $\alpha 2 \beta 1 \gamma 1$ structures ${ }^{8}$. The C2-bound $\gamma 1$-subunit conformation closely mimics that of the AMP-bound complex. Despite the considerable chemical differences and different binding orientations between $\mathrm{C} 2$ and AMP the side chains of the $\gamma 1$-subunit residues are remarkably similar in conformation, with the notable exceptions being $\gamma$-Arg70 and $\gamma$-His298 (Supplementary Fig. 4a,b). A region that does distinguish between the two heterotrimers is the nucleotide sensing $\alpha$-subunit RIM2 motif ${ }^{1,6,8}$. Strong electron density was observed for the $\alpha$-RIM2 in the AMP-bound heterotrimer, but only very weak electron density was observed for $\alpha$-RIM2 in the corresponding C2-bound heterotrimer and could therefore not

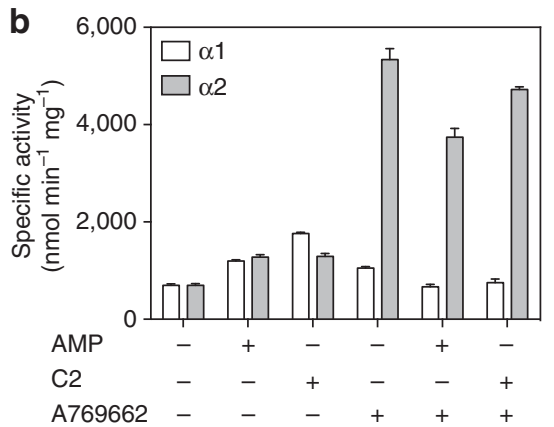

Figure 2 | C2/A769662 synergistic activation of AMPK. Wild-type AMPK $\alpha 1$ and $\alpha 2$ were pre-treated with (a) $\lambda$-phosphatase or (b) CaMKK2, and assayed in the absence/presence of AMP $(100 \mu \mathrm{M})$ and C2 $(0.1 \mu \mathrm{M})$, either individually or in combination with A769662 (20 $\mu$ M). Results from triplicate experiments are presented as mean specific activity ( $\mathrm{nmol} \mathrm{min}^{-1} \mathrm{mg}^{-1} \pm$ s.e.m.), with statistical tests conducted using one-way ANOVA Sidak's multiple comparisons test. Symbols $\S, \dagger$ and \#; $P<0.01$ indicate significant increase in activity compared with A769662, C2 and AMP controls, respectively, while NS indicates that there was no significant difference between the co-incubated samples and their individual control samples. 
Table 1 | Table summarizing basal activity of AMPK $\alpha 1$ and $\alpha 2$ and their fold activation to control, calculated from data in Fig. 2.

\begin{tabular}{|c|c|c|c|c|c|c|c|}
\hline Kinase & Treatment & Basal activity (nmol $\left.\min ^{-1} \mathrm{mg}^{-1}\right)( \pm$ s.e.m.) & \multicolumn{5}{|c|}{ Fold activation to control ( \pm s.e.m.) } \\
\hline$\alpha 1$ & $\begin{array}{c}\lambda \text {-Phosphatase } \\
\text { CaMKK2 }\end{array}$ & $\begin{array}{c}0.6 \pm 0.2 \\
699.8 \pm 26.1\end{array}$ & $\begin{array}{l}7.7 \pm 0.3 \\
1.7 \pm 0.1\end{array}$ & $\begin{array}{l}8.8 \pm 0.3 \\
2.5 \pm 0.1\end{array}$ & $\begin{array}{c}91.8 \pm 5.9 \\
1.5 \pm 0.1\end{array}$ & $\begin{array}{c}1100 \pm 77 \\
1.0 \pm 0.1\end{array}$ & $\begin{array}{c}1040 \pm 43 \\
1.1 \pm 0.1\end{array}$ \\
\hline$\alpha 2$ & $\begin{array}{c}\lambda \text {-Phosphatase } \\
\text { CaMKK2 }\end{array}$ & $\begin{array}{c}1.2 \pm 0.4 \\
697.6 \pm 36.6\end{array}$ & $\begin{array}{l}3.9 \pm 0.5 \\
1.8 \pm 0.1\end{array}$ & $\begin{array}{l}2.1 \pm 0.3 \\
1.9 \pm 0.1\end{array}$ & $\begin{array}{l}1.1 \pm 0.1 \\
7.7 \pm 0.3\end{array}$ & $\begin{array}{r}6.1 \pm 0.1 \\
5.4 \pm 0.5\end{array}$ & $\begin{array}{l}1.2 \pm 0.2 \\
6.8 \pm 0.3\end{array}$ \\
\hline
\end{tabular}

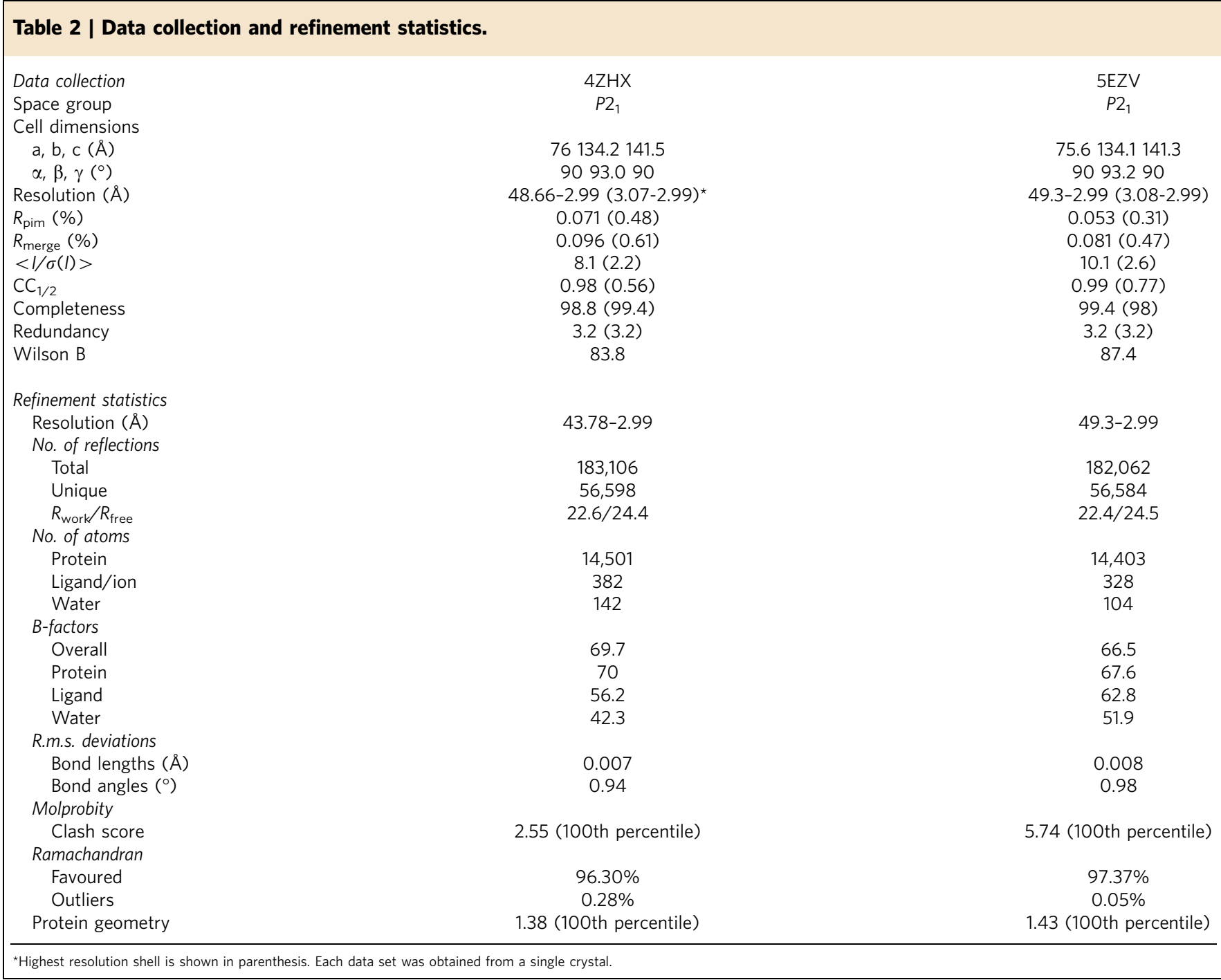

be included in this model (Supplementary Fig. 3b). This is a C2 specific effect as the previously published AMPK structure 4CFE (crystallized in the same space group (P21) and solved to an equivalent resolution of $3.02 \AA$ ) revealed strong electron density for both AMP-bound heterotrimers in the asymmetric unit ${ }^{8}$. Importantly, these data are consistent with the $\alpha 2$-subunit RIM2 being incompletely engaged with the $\gamma$-subunit when $\mathrm{C} 2$ is bound.

The two C2 molecules occupy the interface between the CBS-binding sites 1, 3 and 4, with the phosphate group of C2 occupying a similar position to that of AMP-bound phosphate group in sites 1 and 4 (Fig. 3b). For this reason we have named specific C2-binding sites $\gamma$-pSite- 1 and $\gamma$-pSite- 4 , referring to the equivalent AMP phosphate group position (Fig. 3b,c).
In both sites the $\mathrm{C} 2$ molecule makes six protein-mediated hydrogen bond contacts, while C2 in $\gamma$-pSite-4 makes an additional $\pi$-stacking interaction with $\gamma$-His298 (Fig. 3c). All of the residues that contact $\mathrm{C} 2$ are shared with $\mathrm{AMP}$, yet the binding constant for $\mathrm{C} 2$ is $>100$-fold higher than that for AMP ${ }^{15,16}$. The basis of C2's increased affinity is not obvious to us.

Mutational analysis of the C2-binding site. To validate the C2-binding sites, we expressed three AMPK $\gamma 1$ mutants (T89E, $\mathrm{H} 151 \mathrm{E}$ and $\mathrm{H} 298 \mathrm{E}$ ) in COS7 mammalian cells and measured their sensitivity to C2 or AMP. We initially found that all the $\gamma 1$ mutants displayed increased but variable Thr172 phosphorylation and basal activities compared with the wild-type (WT) 

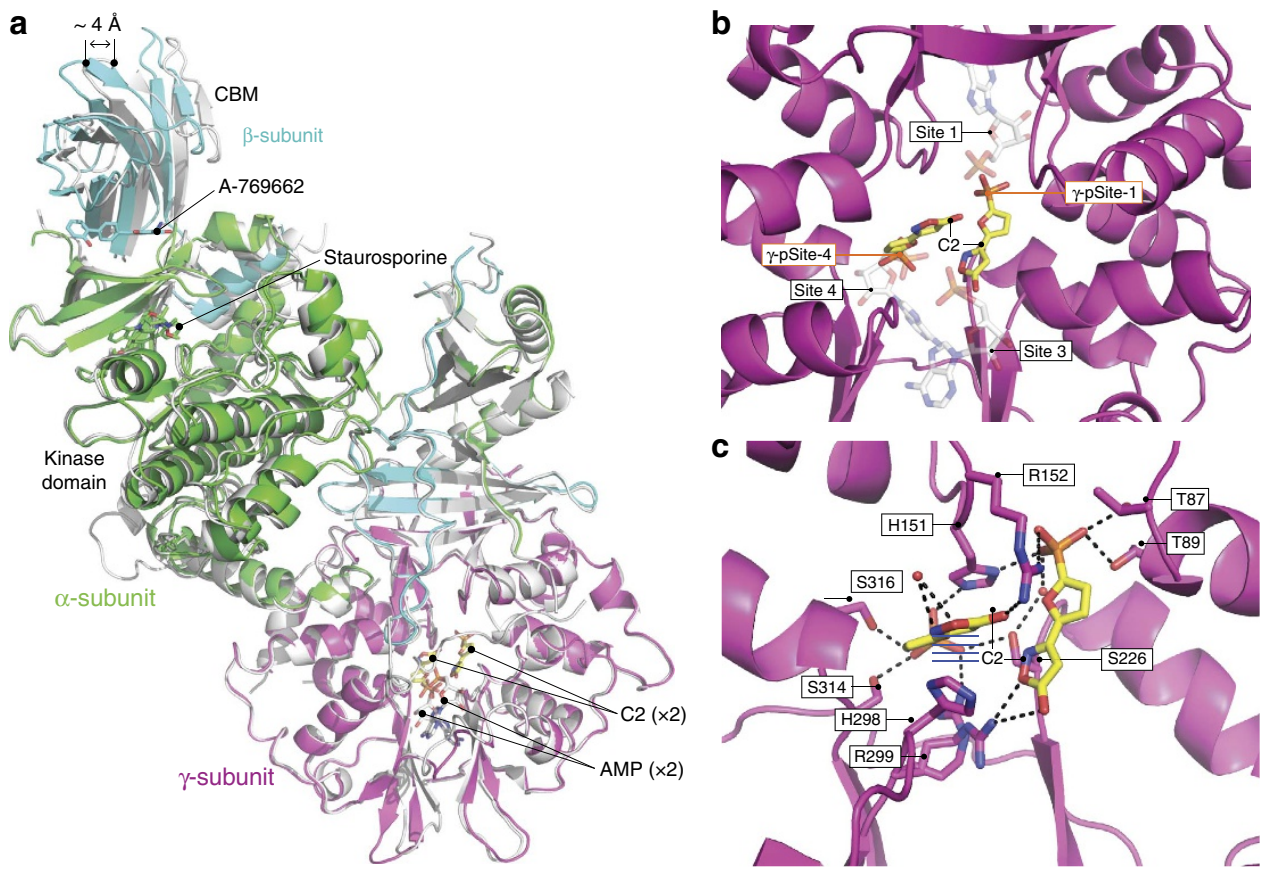

Figure 3 | C2 binds the $\gamma$-subunit of AMPK. (a) Cartoon representation of the AMPK heterotrimer ( $\alpha 2 \beta 1 \gamma 1$ ) bound to C2 (yellow sticks) in the $\gamma$-subunit (magenta), Staurosporine bound to the $\alpha$-subunit (green) and A769662 bound at the ADaM binding site at the interface of the $\alpha$-kinase domain and the CBM of the $\beta$-subunit (cyan) superimposed with the AMP-bound heterotrimer (white). Arrow shows the approximate distance between equivalent $\beta C B M$ residues in the C2-bound and AMP-bound heterotrimers. (b) The position of C2 with respect to CBS sites, with AMP superimposed (from PDB 4CFE). (c) AMPK interactions (black dashed lines and blue parallel lines for $\pi$ stacking) with C2 (yellow sticks). Key residues (labelled) are modelled as sticks. Note that $\gamma$-Arg299 was modeled in two discrete conformations.

$\gamma 1$-containing AMPK complexes. We therefore co-expressed the mutants with the phospho-mimetic AMPK $\alpha 1(\mathrm{~T} 172 \mathrm{D})$, which is more amenable to studying the effect of these $\gamma$ mutations on allosteric activation (Supplementary Fig. 2b) ${ }^{5,21}$. C2 and AMP activated the AMPK $\alpha 1$ (T172D) $\beta 1 \gamma 1$ enzyme to a similar extent $(\approx 8$ - and 10-fold, respectively; Fig. 4a). The $\gamma$-T89E and $\gamma$-H298E mutants had increased basal activities $(9.5 \pm 0.2$ and $11.4 \pm 0.2 \mathrm{nmol} \mathrm{min}^{-1} \mathrm{mg}^{-1}$ lysate for $\gamma$-T89E and $\gamma$-H298E, respectively) in the absence of $\mathrm{C} 2$ and AMP compared with $\gamma 1$ WT ( $1.4 \pm 0.1 \mathrm{nmol} \mathrm{min}^{-1} \mathrm{mg}^{-1}$ lysate), and were insensitive to further activation by either C2 or AMP (Fig. 4a). It is possible that substituting for a negatively charged glutamate residue at the T89 and $\mathrm{H} 298$ positions mimics the effect of the phosphate groups of AMP or C2, resulting in constitutive activation. This would also explain the increased Thr172 phosphorylation observed with these mutants. The $\gamma \mathrm{H} 151 \mathrm{E}$ mutation completely abolished AMPK activation by C2 (Fig. $4 \mathrm{a}, \mathrm{c}$ ), while only partially reducing AMP-dependence and sensitivity compared to the wild-type control (Fig. 4a,b).

C2-induced conformational rearrangements of the $\boldsymbol{\gamma}$-subunit. A superposition of the two AMPK heterotrimers reveals two major side-chain conformational changes between AMP and C2 binding. Firstly, the imidazole ring of the aforementioned $\gamma$-His298 has flipped $180^{\circ}$ and rotated at the $C \beta>90^{\circ}(\sim 6.0 \AA)$ to form a $\pi$-stacking interaction with $\mathrm{C} 2$ in $\gamma$-pSite- 4 while simultaneously blocking site 3 , mimicking ATP-bound AMPK $(\mathrm{PDB} 4 \mathrm{EAK})^{3}$ (Supplementary Fig. 4a). The conformational difference of the $\gamma$-His298 side chain is critical for AMP or C2 binding. Secondly, the position of C2 at $\gamma$-pSite- 1 causes the sidechain guanidine group of $\gamma$-Arg70 to shift approximately $4 \AA$ away from the AMP-bound conformation. In this new conformation, $\gamma$-Arg70 would clash with critical AMP-sensing residue $\alpha$-Glu368 from the $\alpha$ RIM2 (Supplementary Fig. $4 \mathrm{~b}$ ).
The clash may cause $\alpha$-Glu368 to lose hydrogen bonds to site 3 AMP-interacting residues $\gamma$-Arg70 and $\gamma$-Lys170, that tether $\alpha$-Glu368 to the $\gamma$-subunit ${ }^{1,6,8}$. In turn, this causes the $\alpha$ RIM2 disengagement. The loss of RIM2 association with the $\gamma$-subunit leads to the loss of protection from $\alpha$-Thr172 dephosphorylation and a concomitant reduction in $\mathrm{Vmax}$ with $\mathrm{C} 2$ as shown by Hunter et al. ${ }^{16}$. In support of this proposal, previous studies have shown that $\alpha$-Glu368 mutation results in a comparable reduction in Vmax with AMP stimulation and $\alpha$-Thr172 dephosphorylation protection $^{6-8}$.

AMPK isoform specificity of C2 allosteric activation. Secondary structure elements of $\alpha$ RIM 2 are crucial for the isoform specificity of C2. Structural comparison of $\alpha 1$ and $\alpha 2$ isoform $\alpha$ RIM2/ $\gamma$-subunit interactions reveal the $\alpha$-helical $\alpha$ 1RIM2 (PDB 4CFH) ${ }^{1}$ forms a strong interaction (three hydrogen bonds and six saltbridges $)^{22}$ with the $\gamma$-subunit in a groove between $\gamma$-helix $\alpha 10$ and $\gamma$-helix $\alpha 4$, formed upon AMP or C2 binding (Supplementary Fig. 3b,c). In contrast, $\alpha 2$ isoforms have a loop motif (PDB 4ZHX and $4 \mathrm{CFE})^{8}$ that forms a single hydrogen bond and four salt-bridges $^{22}$ with the $\gamma$-subunit (Supplementary Fig. 3b,c). We propose the weaker interacting $\alpha 2$ isoform $\alpha$ RIM2 is incompletely engaged upon C2 binding relative to the $\alpha 1$ isoform, resulting in the $\alpha$-isoform specificity for allosteric and synergistic activation and protection from $\alpha$-Thr172 dephosphorylation. We solved the $\mathrm{X}$-ray crystal structure of a heterotrimeric $\alpha 2 / \alpha 1$ RIM chimaera, $\alpha 2(1-347) / \alpha 1(349-401) / \alpha 2(397-$ end) $\beta 1 \gamma 1$ (which has previously been shown to completely restore maximal allosteric activation and C2-mediated protection of $\alpha$-Thr172 dephosphorylation ${ }^{16}$ ), co-crystallized with C2 to $2.99 \AA$ (pdb: 5EZV; Table 2). Unlike the aforementioned crystal structure (4ZHX), $\alpha 2 / \alpha 1$ RIM swap chimaera was crystallized in the absence of AMP, revealing two C2-bound AMPK heterotrimers in the asymmetric unit. Both of the C2-bound heterotrimers $(5 \mathrm{EZV})$ closely resembled the 
previous C2-bound heterotrimer (molecule $14 \mathrm{ZHX}$ ), with an r.m.s deviation of 0.520 over $869 \mathrm{C} \alpha$ atoms (molecule 1 5EZV). The C2 molecules occupy the same binding space and $\gamma$-subunit
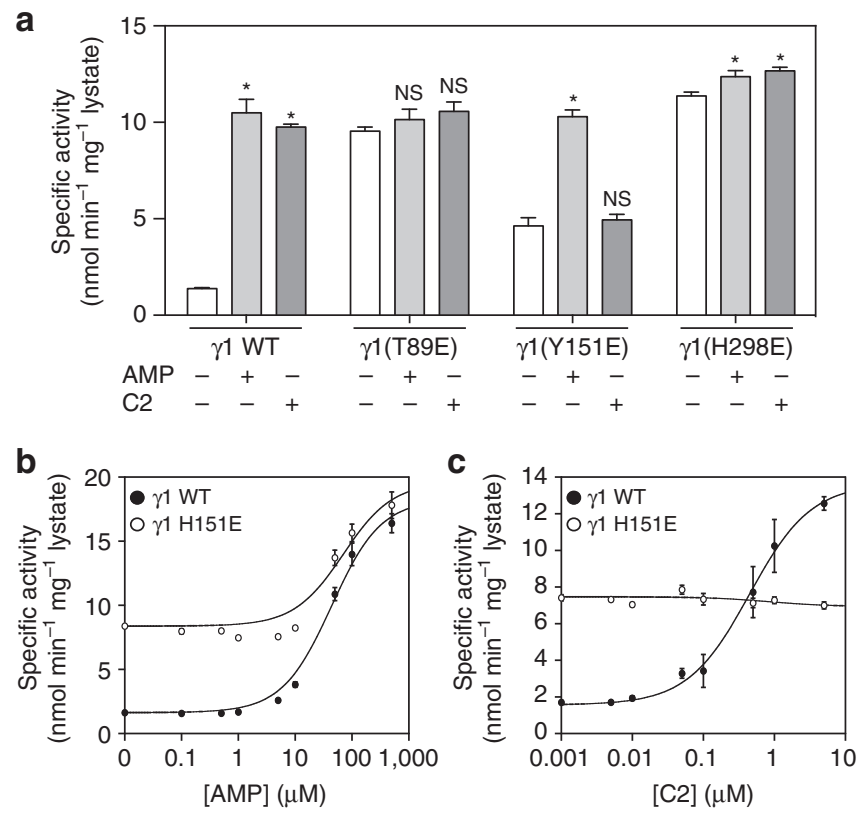

Figure 4 | Effect of AMPK $\gamma$-subunit mutations on C2 activity. AMPK $\alpha 1 \beta 1 \gamma 1$ phospho-mimetic T172D and their respective $\gamma$ mutants T89E, $\mathrm{H} 151 \mathrm{E}$, and H298E were expressed as FLAG- $\alpha$ fusion proteins in COS7 mammalian cells. (a) Activities of AMPK mutants were tested in the absence or presence of C2 $(5 \mu \mathrm{M})$ or AMP $(100 \mu \mathrm{M})$. Results from four independent experiments are presented as specific activity (nmol $\mathrm{min}^{-1} \mathrm{mg}^{-1}$ lysate \pm s.e.m.), with statistical tests conducted using one-way ANOVA. ${ }^{\star} P<0.05$ indicates significant increase in activity compared with untreated control. (b) AMP dose-response of AMPK $\alpha 1-T 172 \mathrm{D} \gamma 1$ wild-type and $\gamma 1-\mathrm{H} 151 \mathrm{E}$ mutants $(0-500 \mu \mathrm{M})$. The data was plotted as AMPK activity ( $\mathrm{nmol} \mathrm{min}^{-1} \mathrm{mg}^{-1}$ lysate \pm s.e.m.) versus [AMP] $(\mu \mathrm{M})$ from four independent experiments. (c) C2 dose-response of AMPK $\alpha 1-T 172 \mathrm{D} \gamma 1$ wild-type and $\gamma 1-\mathrm{H} 151 \mathrm{E}$ mutants $(0-5 \mu \mathrm{M})$. Curve was plotted as AMPK activity ( $\mathrm{nmol} \mathrm{min}^{-1} \mathrm{mg}^{-1}$ lysate \pm s.e.m.) versus $[C 2](\mu \mathrm{M})$ from four independent experiments. The values for $\mathrm{EC}_{50}$ and fold stimulation were calculated based on fitting the data to the equation: Activity $=$ Basal $+((($ Fold stimulation $\times$ Basal $)-$ Basal $\times[C 2]) /$ $\left.\left(\left(E_{50}\right)+[C 2]\right)\right)$. residue interactions as the $\mathrm{AMP} / \mathrm{C} 2$-bound structures (4ZHX). Importantly the structure reveals density for seven $\alpha 1$ RIM 2 residues (Fig. 5), including the main-chain of the nucleotide sensing $\alpha$-E364 ( $\alpha 2$ E368), further supporting our proposal that the $\alpha 1$ RIM2 interacts more strongly with the $\gamma$-subunit. Interestingly there was weak density for the carboxyl group of the E364 side-chain, possibly indicating a transient interaction with the $\gamma$-subunit residue R70.

\section{Discussion}

In this study we showed that two topographically disparate drug sites on AMPK, one located at the classic $\alpha$-kinase domain/ $\beta$-CBM interface (ADaM site) and the other located within the solvent-accessible core of the $\gamma$-subunit ( $\gamma$-pSite- $1 / \gamma-$ pSite- 4$)$, can be exploited to achieve synergistic activation of unphosphorylated a1-AMPK independent of AMP and upstream phosphorylation events. This provides a major advance to previous demonstrations of AMPK synergistic activation that have been dependent on AMP. Importantly, identification of C2-binding sites on the $\gamma$-subunit represents an entirely unexplored dimension for future rational drug design.

\section{Methods}

Reagents. Antibodies for pan AMPK $\alpha$ (\#2793), FLAG (\#2368) and HA (\#2367); phosphospecific antibodies for $\alpha$-pThr172 (\#2535) and $\beta$-pSer108 (\#4181), and $\lambda$-phosphatase (\#P0753) were from Cell Signaling Technology. $\beta 1$ antibody (\#ab58175) and A769662 (\#ab120335) were from Abcam. Anti-rabbit and antimouse IgG secondary antibodies fluorescently labelled with IR680 or IR800 dye, respectively, were from LI-COR Biosciences. Glutathione Sepharose 4B was from GE Life Sciences. FLAG peptide (DYKDDDK) was provided by GL Biochem (Shanghai), purified by reversed-phase chromatography and stored as lyophilized powder. All other reagents were purchased from Sigma.

Constructs for crystallization. All mutants were generated using QuikChange Site-Directed Mutagenesis Kits (Stratagene), and constructs were sequence verified. Escherichia coli-expressed AMPK preparations were mass verified by time-of-flight (TOF) mass spectrometry. Heterotrimeric human AMPK His6- $\alpha 2 \beta 1 \gamma 1$ was expressed in E. coli strain Rosetta (DE3) using the pETDuet ${ }^{\mathrm{TM}}-1$ expression system (Novagen) as previously described ${ }^{13}$. Briefly, cDNAs for $\alpha 2$ and $\gamma 1$ were sequentially inserted into pETDuet ${ }^{\mathrm{TM}}-1$ multiple cloning sites (MCS) 1 (BamH1/ Not1) and 2 (MfeI/XhoI), respectively, resulting in incorporation of an N-terminal hexahistidine tag onto $\alpha 2$. cDNA for $\beta 1$ was inserted into pRSFDuet ${ }^{\mathrm{TM}_{-1}}$ MCS1 (NcoI/ BamHI). Heterotrimeric human AMPK $\alpha 2 / \alpha 1$ RIM chimaera ( $\alpha 2(1-347) /$ $\alpha 1(349-401) / \alpha 2$ (397-end) $\beta 1 \gamma 1)^{16}$ was expressed in E. coli strain Rosetta (DE3) using the pETDuet ${ }^{\mathrm{TM}}-1$ and pCOLADuet ${ }^{\mathrm{TM}}$ (Novagen). cDNAs for $\alpha 2 / 1$ chimaera and $\gamma 1$ were sequentially inserted into pETDUET $^{\mathrm{TM}}-1$ multiple cloning sites (MCS) 1 (BamHI/Not1) and 2 (MfeI/Xhol), respectively, resulting in incorporation of an $\mathrm{N}$-terminal hexahistidine tag onto $\alpha 2 / 1$ chimaera. cDNA for $\beta 1$ was inserted into pCOLADuet ${ }^{\mathrm{TM}}-1$ MCS1 (NcoI/ BamHI).

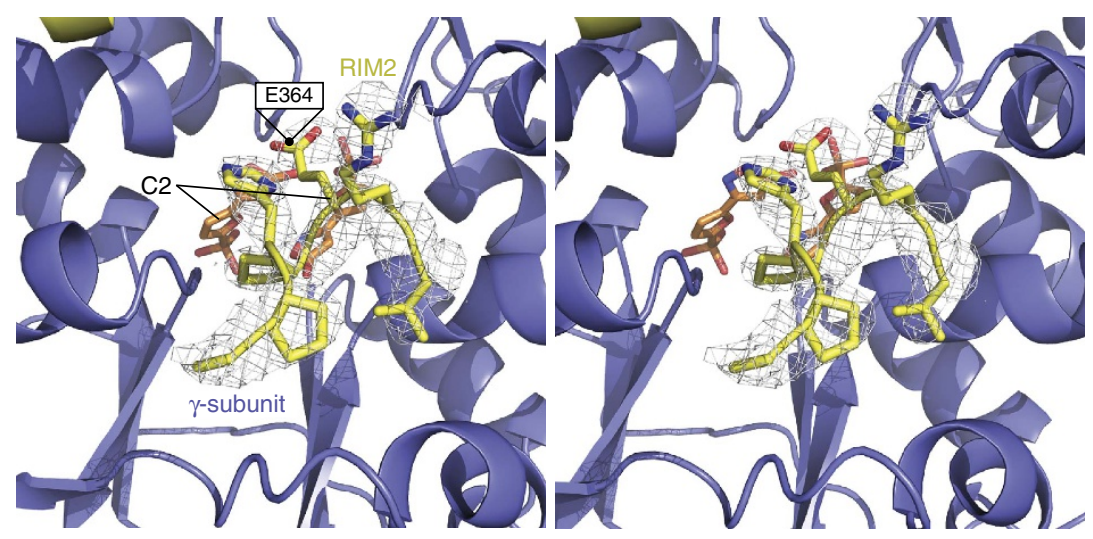

Figure 5 | Omit map of the $\alpha \mathbf{1 R I M 2}$ with $\mathbf{C 2}$ bound in the $\boldsymbol{\gamma}$-subunit. Stereo $2 F_{o}-F_{c}$ omit map of the $\alpha 1 R I M 2$ (yellow) from the heterotrimeric human AMPK RIM swap chimaera ( $\alpha 2$ 1-347/ $\alpha 1$ 349-401/ $\alpha 2$ 397-end $\beta 1 \gamma 1$ ) bound to C2, contoured at $1 \sigma$. Continuous density (white mesh) can be seen for seven residues of the $\alpha 1$ RIM2, including E364 ( $\alpha 2$ E368). Weak density for the side-chain carboxyl of E364 suggests some interaction with C2 (orange sticks) bound $\gamma$-subunit (blue). 
Protein expression and purification for crystallization. Expression cultures were grown in Luria-Bertani broth and induced at $16^{\circ} \mathrm{C}$ with $0.25 \mathrm{mM}$ isopropyl $\beta$-D-1-thiogalactopyranoside, before overnight incubation. Cells were lysed using a precooled EmulsiFlex-C5 homogenizer (Avestin) and AMPK purified using Nickel Sepharose and size exclusion chromatography in $50 \mathrm{mM}$ Tris- $\mathrm{HCl}, \mathrm{pH} 8.0$, $150 \mathrm{mM} \mathrm{NaCl}, 2 \mathrm{mM}$ Tris(2-carboxyethyl)phosphine buffer. AMPK was phosphorylated by incubation with CaMKK2 (expressed in Sf21 cells as a $\mathrm{C}$-terminal FLAG fusion and purified on FLAG monoclonal antibody-coupled affinity resin $^{2}$ ) in the presence of $2.5 \mathrm{mM} \mathrm{MgCl}_{2}, 0.5 \mathrm{mM}$ ATP and $0.5 \mathrm{mM}$ AMP $\left(1 \mathrm{~h}, 22^{\circ} \mathrm{C}\right)$. Protein was re-purified using size exclusion chromatography, concentrated to $\sim 10 \mathrm{mg} \mathrm{ml}^{-1}$ and flash frozen in liquid nitrogen before storage at $-80^{\circ} \mathrm{C}$ (storage buffer: $50 \mathrm{mM}$ Tris- $\mathrm{HCl}, \mathrm{pH} 8.0,150 \mathrm{mM} \mathrm{NaCl}, 2 \mathrm{mM}$ Tris(2-carboxyethyl)phosphine).

Crystallization. Full-length myristoylated and phosphorylated $\alpha 2 \beta 1 \gamma 1$ $\left(4 \mathrm{mg} \mathrm{ml}^{-1}\right)$ was mixed with a twofold molar excess of C2, AMP and S108tide (synthetic peptide: $\beta 1(102-117) \mathrm{R}^{115-117}, \mathrm{NH}_{2}$-KLPLTRSHNNFVARRR-COOH) and a 1:1 molar ratio of Staurosporine and A769662, while full-length phosphorylated RIM swap chimaera ( $\alpha 21-347 / \alpha 1$ 349-401/ $\alpha 2$ 397-end $\beta 1 \gamma 1)^{16}$ $\left(4 \mathrm{mg} \mathrm{ml}^{-1}\right)$ was mixed with a sixfold molar excess of C2, twofold molar excess of S108tide and a 1:1 molar ratio of Staurosporine and A769662. Crystals from both protein preparations were grown following the previously described $\operatorname{method}^{8}$. Briefly, protein was mixed equally at room temperature with a reservoir solution containing 8\% PEG 3350, $0.1 \mathrm{M} \mathrm{MgCl}_{2}, 1.0 \%$ glucose, $0.001 \%$ cocamidopropyl betaine and $0.1 \mathrm{M}$ imidazole ( $\mathrm{pH}$ 6.2). Protein crystals were incubated with reservoir solution containing an additional 5\% glycerol, 5\% PEG 400, 5\% MPD, 5\% sucrose, $5 \%$ sorbitol and $5 \%$ ethylene glycol for $\sim 2 \mathrm{~min}$ before flash-cooling in liquid nitrogen. Data were collected at the Australian Synchrotron MX2 beamline, Melbourne, Australia. Data were processed and integrated using XDS $^{23}$ and scaled using AIMLESS from the CCP4 suite ${ }^{24}$. The structure was solved by molecular replacement using Phaser from the $\mathrm{CCP} 4$ suite $^{25}$ and $4 \mathrm{CFE}^{8}$ as the search model. Iterative rounds of model building and refinement were performed using $\operatorname{Coot}^{26}$ and Buster (https://www.globalphasing.com/buster/) ${ }^{27}$ respectively. C2 molecular coordinates and restraints were generated using the PRODRG web-server ${ }^{28}$. Omit maps were calculated using Sfcheck in the CCP4 suite ${ }^{29}$. Structure validation and protein-C2 interactions was performed using Molprobity ${ }^{30}$, figures were created using Pymol (https://www.pymol.org/). Data collection and refinement statistics are listed in Table 2.

\section{Protein expression and purification for AMPK enzyme assays. All mutants} were generated using QuikChange Site-Directed Mutagenesis Kits (Stratagene), and constructs were sequence verified. Heterotrimeric AMPK was expressed in COS7 mammalian cells by transient transfection, using FuGENE HD (Promega) transfection reagent according to manufacturer's protocol. Transfected constructs included N-terminal GST- $\alpha 1$ and $\alpha 2$ fusions $^{2}$ (pDEST27 expression vector), C-terminal FLAG- $\alpha 1$ and $\alpha 2$ fusions (cloned into pcDNA3.1 expression vector using XhoI/EcoRI $(\alpha 1)$ or XhoI/HindIII $(\alpha 2)$ restriction sites, C-terminal myc- $\beta 1$ and $\beta 2$ fusions ${ }^{31}$ (pcDNA3.1 expression vector) and N-terminal HA- $\gamma 1, \gamma 2$ and $\gamma 3$ fusions $^{32}$ (pMT2 expression vector). To assess C2 $\gamma$ isoform selectivity and C2 kinetics (Fig. 1): GST- $\alpha 1 \beta 1 / 2 \gamma 1 / 2 / 3$ AMPK complexes were isolated from transfected lysates by immobilization on Glutathione Sepharose 4B (GE Life Sciences) prior to SAMS activity assay ${ }^{2}$. To assess C2/A769662 synergy (Fig. 2): GST- $\alpha 1 \beta 1 \gamma 1$ and GST- $\alpha 2 \beta 1 \gamma 1$ AMPK complexes were immobilized on Glutathione Sepharose $4 \mathrm{~B}$ and incubated with either $\lambda$-phosphatase $(50 \mathrm{mM}$ Tris- $\mathrm{HCl}$ pH 7.4, $150 \mathrm{mM} \mathrm{NaCl}, 10 \%$ glycerol, $0.01 \%$ Tween-20 (buffer A) supplemented with $2 \mathrm{mM} \mathrm{MnCl}_{2}$ ) or CaMKK2 (buffer A supplemented with $2 \mathrm{mM}$ $\left.\mathrm{MgCl}_{2}, 200 \mu \mathrm{M} \mathrm{ATP}\right)\left(1 \mathrm{~h}, 22^{\circ} \mathrm{C}\right)$ prior to extensive washes with buffer A and elution in buffer A supplemented with $20 \mathrm{mM}$ glutathione. The levels of $\alpha$-pThr172 and $\beta$-pSer108 of treated AMPK complexes were assessed by immunoblot as described previously ${ }^{32}$ (Supplementary Fig. 2a). Briefly, membranes were simultaneously probed overnight at $4{ }^{\circ} \mathrm{C}$ with a phosphospecific primary antibody for $\alpha$-pThr172 (1:2,000 dilution in phosphate buffered saline with $0.1 \%$ Tween-20 $(\mathrm{v} / \mathrm{v})(\mathrm{PBS}-\mathrm{T}))$ or $\beta$-pSer108 (1:2,000 dilution in PBS-T) labelled with the fluorescent dye IR680 (LI-COR Biosciences) and probed for $1 \mathrm{~h}$ at room temp with pan $\alpha$ primary antibodies (1:1,000 dilution in PBS-T) or $\beta 1$ primary antibodies (1:1,000 dilution in PBS-T) fluorescently labelled with IR800. Immunoreactive bands were visualized on an Odyssey membrane imaging system (LI-COR Biosciences) and densitometry analyses performed using integrated software. To evaluate the impact of $\gamma$-mutation on C2/AMP activity (Fig. 4): FLAG$\alpha 1$ (T172D) $\beta 1 \gamma 1$ (wild-type and indicated mutants) AMPK complexes were immobilized on anti-FLAG M2 affinity Agarose gel (Supplementary Fig. 2b) before SAMS activity assay. The expression levels of the $\gamma$-mutants were assessed by immunoblot. Membrane was probed overnight at $4{ }^{\circ} \mathrm{C}$ with a FLAG primary antibody (1:2,000 dilution in PBS-T) labelled with the fluorescent dye IR680 (LI-COR Biosciences) and probed for $1 \mathrm{~h}$ at room temp with HA primary antibodies (1:1,000 dilution in PBS-T) fluorescently labelled with IR800 and analysed as above.

AMPK activity assay. AMPK activity assay was conducted as described previously ${ }^{13}$. Briefly, AMPK complexes were purified as above and washed three times with a $40: 1 \mathrm{v} / \mathrm{v}$ ratio of assay buffer/resin (assay buffer: $50 \mathrm{mM}$ Hepes $\mathrm{pH} 7.4$,
$1 \mathrm{mM}$ DTT and $0.02 \%$ Brij-35). Reaction mixtures $(25 \mu \mathrm{l})$ containing $100 \mu \mathrm{M}$ SAMS synthetic peptide (sequence: $\mathrm{NH}_{2}$-HMRSAMSGLHLVKRR-COOH), $5 \mathrm{mM}$ $\mathrm{MgCl}_{2}, 200 \mu \mathrm{M}\left[\gamma_{-}{ }^{32} \mathrm{P}\right]$-ATP were incubated AMPK for $10 \mathrm{~min}$ at $30^{\circ} \mathrm{C}$ in the absence or presence of various C2 concentrations. AMP $(100 \mu \mathrm{M})$ and/or A769662 $(20 \mu \mathrm{M})$ were included as appropriate positive controls. After $10 \mathrm{~min}$, phosphotransferase activity was quenched by spotting onto P81 ion-exchange chromatography paper (Whatman, GE Healthcare, Parramatta, NSW) followed by repeated washes in $1 \% \mathrm{H}_{3} \mathrm{PO}_{4}$ (ref. 33). The level of ${ }^{32} \mathrm{P}$-transfer to the SAMS peptide was determined by liquid scintillation counting (Perkin Elmer, Melbourne, VIC). Statistical tests were conducted using analysis of variance (ANOVA). The values for $\mathrm{EC}_{50}$ and fold stimulation were calculated based on the equation: Activity $=$ Basal $+\left(((\right.$ Fold stimulation $\times$ Basal $)-$ Basal $\left.\times[\mathrm{C} 2]) /\left(\left(\mathrm{EC}_{50}\right)+[\mathrm{C} 2]\right)\right)$. Curve fitting was performed using KaleidaGraph software.

\section{References}

1. Xiao, B. et al. Structure of mammalian AMPK and its regulation by ADP. Nature 472, 230-233 (2011).

2. Oakhill, J. S. et al. beta-Subunit myristoylation is the gatekeeper for initiating metabolic stress sensing by AMP-activated protein kinase (AMPK). Proc. Natl Acad. Sci. USA 107, 19237-19241 (2010).

3. Chen, L. et al. AMP-activated protein kinase undergoes nucleotide-dependent conformational changes. Nat. Struct. Mol. Biol. 19, 716-718 (2012).

4. Oakhill, J. S. et al. AMPK is a direct adenylate charge-regulated protein kinase. Science 332, 1433-1435 (2011).

5. Gowans, G. J., Hawley, S. A., Ross, F. A. \& Hardie, D. G. AMP is a true physiological regulator of AMP-activated protein kinase by both allosteric activation and enhancing net phosphorylation. Cell Metab. 18, 556-566 (2013).

6. Chen, L. et al. Conserved regulatory elements in AMPK. Nature 498, E8-E10 (2013).

7. Li, X. et al. Structural basis of AMPK regulation by adenine nucleotides and glycogen. Cell Res. 25, 398 (2015).

8. Xiao, B. et al. Structural basis of AMPK regulation by small molecule activators Nat. Commun. 4, 3017 (2013).

9. Giordanetto, F. \& Karis, D. Direct AMP-activated protein kinase activators a review of evidence from the patent literature. Expert Opin. Ther. Pat. 22, 1467-1477 (2012).

10. Calabrese, M. F. et al. Structural basis for AMPK activation: natural and synthetic ligands regulate kinase activity from opposite poles by different molecular mechanisms. Structure. 22, 1161-1172 (2014).

11. Langendorf, C. G. \& Kemp, B. E. Choreography of AMPK activation. Cell Res. 25, 5-6 (2015).

12. Hawley, S. A. et al. The ancient drug salicylate directly activates AMP-activated protein kinase. Science 336, 918-922 (2012).

13. Scott, J. W. et al. Small molecule drug A-769662 and AMP synergistically activate naive AMPK independent of upstream kinase signaling. Chem. Biol. 21, 619-627 (2014).

14. Sanders, M. J. et al. Defining the mechanism of activation of AMP-activated protein kinase by the small molecule A-769662, a member of the thienopyridone family. J. Biol. Chem. 282, 32539-32548 (2007).

15. Gomez-Galeno, J. E. et al. A potent and selective AMPK activator that inhibits de novo lipogenesis. ACS Med. Chem. Lett. 1, 478-482 (2010).

16. Hunter, R. W. et al. Mechanism of action of compound-13: an alpha1-selective small molecule activator of AMPK. Chem. Biol. 21, 866-879 (2014).

17. Ross, F., Jensen, T. E. \& Hardie, D. G. Differential regulation by AMP and ADP of AMPK complexes containing different gamma subunit isoforms. Biochem. J. 473, 189-199 (2016).

18. Barnes, B. R. et al. The 5'-AMP-activated protein kinase gamma3 isoform has a key role in carbohydrate and lipid metabolism in glycolytic skeletal muscle. J. Biol. Chem. 279, 38441-38447 (2004).

19. Rajamohan, F. et al. Escherichia coli expression, purification and characterization of functional full-length recombinant alpha2beta2gamma3 heterotrimeric complex of human AMP-activated protein kinase. Protein Expr. Purif. 73, 189-197 (2010).

20. Kornev, A. P. \& Taylor, S. S. Defining the conserved internal architecture of a protein kinase. Biochim. Biophys. Acta 1804, 440-444 (2010).

21. Stein, S. C., Woods, A., Jones, N. A., Davison, M. D. \& Carling, D. The regulation of AMP-activated protein kinase by phosphorylation. Biochem. J. 345(Pt 3): 437-443 (2000).

22. Krissinel, E. \& Henrick, K. Inference of macromolecular assemblies from crystalline state. J. Mol. Biol. 372, 774-797 (2007).

23. Kabsch, W. XDS. Acta Crystallogr. D Biol. Crystallogr. 66, 125-132 (2010).

24. Evans, P. R. \& Murshudov, G. N. How good are my data and what is the resolution? Acta Crystallogr. D Biol. Crystallogr. 69, 1204-1214 (2013).

25. McCoy, A. J. et al. Phaser crystallographic software. J. Appl. Crystallogr. 40, 658-674 (2007).

26. Emsley, P., Lohkamp, B., Scott, W. G. \& Cowtan, K. Features and development of Coot. Acta Crystallogr. D Biol. Crystallogr. 66, 486-501 (2010).

27. Bricogne, G. et al. BUSTER, Version 2.10.0 (Global Phasing Ltd., Cambridge, United Kingdom, 2011). 
28. Schuttelkopf, A. W. \& van Aalten, D. M. PRODRG: a tool for high-throughput crystallography of protein-ligand complexes. Acta Crystallogr. D Biol. Crystallogr. 60, 1355-1363 (2004).

29. Vaguine, A. A., Richelle, J. \& Wodak, S. J. SFCHECK: a unified set of procedures for evaluating the quality of macromolecular structure-factor data and their agreement with the atomic model. Acta Crystallogr. D Biol. Crystallogr. 55, 191-205 (1999).

30. Chen, V. B. et al. MolProbity: all-atom structure validation for macromolecular crystallography. Acta Crystallogr. D Biol. Crystallogr. D66, 12-21 (2010).

31. Scott, J. W. et al. Inhibition of AMP-activated protein kinase at the allosteric drug-binding site promotes islet insulin release. Chem. Biol. 22, 705-711 (2015).

32. Scott, J. W. et al. Thienopyridone drugs are selective activators of AMP-activated protein kinase betal-containing complexes. Chem. Biol. 15, 1220-1230 (2008).

33. Glass, D. B., Masaracchia, R. A., Feramisco, J. R. \& Kemp, B. E. Isolation of phosphorylated peptides and proteins on ion exchange papers. Anal. Biochem. 87, 566-575 (1978)

\section{Acknowledgements}

This research was partly undertaken on the MX2 beamline at the Australian Synchrotron, Victoria, Australia and we thank the beamline staff for their assistance. This work was supported by grants and fellowships from the Australian Research Council (ARC) and the National Health and Medical Research Council (NHMRC).

Supported in part by the Victorian Government's Operational Infrastructure

\section{Author contributions}

C.G.L., K.R.W.N. and B.E.K. designed the study, C.G.L., K.R.W.N., J.W.S., J.S.O., N.X.Y.L. and S.M.A.I. performed the experiments, C.G.L. solved the structures,
K.S. provided reagents and J.S.O., J.W.S., M.A.G. and M.W.P. provided intellectual input and resources. C.G.L., B.E.K. and K.R.W.N. wrote the manuscript. Other co-authors contributed and edited the manuscript.

\section{Additional information}

Accession codes: Coordinates and structure factors have been deposited in the protein data bank with the accession codes 4ZHX and 5EZV.

Supplementary Information accompanies this paper at http://www.nature.com/ naturecommunications

Competing financial interests: The authors declare no competing financial interests.

Reprints and permission information is available online at http://npg.nature.com/ reprintsandpermissions/

How to cite this article: Langendorf, C. G. et al. Structural basis of allosteric and synergistic activation of AMPK by furan-2-phosphonic derivative C2 binding. Nat. Commun. 7:10912 doi: 10.1038/ncomms10912 (2016).

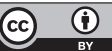

This work is licensed under a Creative Commons Attribution 4.0 International License. The images or other third party material in this article are included in the article's Creative Commons license, unless indicated otherwise in the credit line; if the material is not included under the Creative Commons license, users will need to obtain permission from the license holder to reproduce the material. To view a copy of this license, visit http://creativecommons.org/licenses/by/4.0/ 